\title{
microRNA-15a-5p participates in sepsis by regulating the inflammatory response of macrophages and targeting TNIP2
}

\author{
YUFENG LOU and ZHENRONG HUANG \\ Department of Emergency, The Affiliated Hospital of Medical School of Ningbo University, \\ Ningbo, Zhejiang 315000, P.R. China
}

Received January 24, 2019; Accepted December 9, 2019

DOI: $10.3892 /$ etm.2020.8547

\begin{abstract}
The mortality rate for patients experiencing sepsis is decreasing; however, an effective therapeutic strategy requires further investigation. Increasing evidence has supported the idea that dysregulated microRNAs (miR) participate in the development of sepsis. Meanwhile, macrophages are crucial players in various inflammatory responses and diseases. The objective of the current study was to investigate the associated molecular mechanisms of action of miR-15a-5p on inflammatory responses in lipopolysaccharide (LPS)-stimulated mouse macrophages and the macrophage cell line RAW264.7. RAW264.7 macrophages were stimulated with LPS for $4 \mathrm{~h}$, and ELISAs were subsequently used to measure the expression levels of pro-inflammatory cytokines, including tumor necrosis factor (TNF)- $\alpha$, interleukin (IL)-1 $\beta$ and IL-6, in RAW264.7 macrophages. The expression levels of miR-15a-5p in RAW264.7 macrophages were detected after the stimulation of LPS using reverse transcription quantitative-PCR. The results indicated that the IL-1 $\beta$, IL-6, TNF- $\alpha$ and miR-15a-5p levels were significantly increased compared with the control group. The Target gene prediction database (TargetScan) and dual-luciferase reporter assays were subsequently employed, and TNF- $\alpha$ induced protein 3 -interacting protein 2 (TNIP2) was confirmed as a direct target for miR-15a-5p. Additionally, it was found that the TNIP2 expression levels were decreased in RAW264.7 macrophages following LPS treatment compared with controls. The present study also examined the effects of miR-15a-5p inhibitor on inflammatory cytokine expression levels and the activation of the $N F-\kappa B$ signaling pathway. These results demonstrated that miR-15a-5p inhibitor reduced the secretion of inflammatory cytokines and inhibited $\mathrm{NF}-\kappa \mathrm{B}$ pathway activation by targeting TNIP2. This may be associated with the progression of sepsis. Meanwhile, a LPS-induced
\end{abstract}

Correspondence to: Dr Yufeng Lou, Department of Emergency, The Affiliated Hospital of Medical School of Ningbo University, 247 Renmin Road, Jiangbei, Ningbo, Zhejiang 315000, P.R. China E-mail: luoyufeng0124@163.com

Key words: microRNA-15a-5p, tumor necrosis factor $\alpha$ induced protein 3-interacting protein 2 , macrophages, sepsis mouse model of sepsis was established to examine the regulation of TNIP2 and miR-15a-5p during inflammation. In the animal model, miR-15a-5p inhibitor significantly suppressed the secretion of inflammatory factors. The levels of creatin, blood urea nitrogen, aspartate aminotransferase and alanine aminotransferase in the serum of LPS-treated mice were also found to be decreased in the miR-15a-5p inhibitor treatment group, while the protective effects of miR-15a-5p inhibitor on sepsis were eliminated by TNIP2-small interfering RNA combination therapy. In conclusion, the present findings indicated that miR-15a-5p may be involved in the inflammatory process during sepsis by activating the $\mathrm{NF}-\kappa \mathrm{B}$ pathway and targeting TNIP2. This suggests that miR-15a-5p inhibitor may be a novel anti-inflammatory agent and therapeutic strategy for sepsis.

\section{Introduction}

Sepsis, a severe systemic inflammatory response syndrome (1), results in multiple organ dysfunction, shock and even death (2-4). The high incidence of sepsis in elderly populations results in high mortality and morbidity rates worldwide $(5,6)$. Although great efforts have been made to improve the outcome for the diagnosis and treatment of sepsis, targets for earlier treatment require further investigation $(7,8)$.

MicroRNAs (miRNAs), a class of endogenous non-encoding small molecules of 20-22 nucleotides in length, can regulate the target gene expression by interacting with the 3'-untranslated region (3'-UTR) of target mRNAs $(9,10)$. miRNAs are involved in the regulation of numerous cell functions, including cell growth, differentiation, proliferation and apoptosis (11). Studies have generated promising treatments for complex human diseases by restraining translational, destroying targets and silencing genes $(12,13)$. Moreover, inflammation is a potential sepsis biomarker, and a number of studies have demonstrated that miRNAs serve as important regulators for the inflammatory response by preventing $\mathrm{NF}-\kappa \mathrm{B}$ activation (14). Multiple miRNAs have been confirmed to be dysregulated in sepsis, including miRNA-133a, miRNA-146a, miRNA-297 and miRNA-122, which provide vital references for the diagnosis and treatment of sepsis (15-19). Previous studies have indicated that miRNA (miR)-146a prevents $\mathrm{NF}-\kappa \mathrm{B}$ activation and inflammation by targeting tumor necrosis factor (TNF) receptor-associated factor 6 
and interleukin (IL)-1 receptor-associated kinase $(20,21)$. $\mathrm{NF}-\kappa \mathrm{B}$, a transcription factor, has been reported to regulate the inflammatory response and be activated by several different stimulants, including lipopolysaccharide (LPS). Additionally, $\mathrm{NF}-\kappa \mathrm{B}$ participates in the inflammation process by regulating the expression of inflammatory cytokines and mediator genes (22). Notably, miR-15a-5p is considered a tumor suppressor and serves important roles in a number of cancer types including endometrial cancer (23), human hepatocellular carcinoma (24) and non-small cell lung cancer (25). However, its participation in the progression of sepsis remains unclear.

A previous study has demonstrated that the macrophage-mediated inflammatory response is involved in severe inflammation and the immune suppression of sepsis (20). During sepsis, macrophages mediate the inflammatory response and are heavily involved in the serious inflammation and immune suppression during sepsis. LPS is widely used in both in vitro and in vivo models focusing on inflammation (26). LPS stimulation causes various intracellular activities in macrophages, including the phosphorylation and activation of p65. Phosphorylated (p)-p65 is subsequently transferred into the nucleus where it regulates the expression of genes associated with inflammation (27). TNF- $\alpha$ induced protein 3 -interacting protein (TNIP) 2 is a negative regulator of $\mathrm{NF}-\kappa \mathrm{B}$ signaling. Overexpression of TNIP2 has been reported to suppress $\mathrm{NF}-\kappa \mathrm{B}$ activation (28). However, whether and how miR-15a-5p functions in the inflammatory process in sepsis in vivo and in vitro remains unknown.

In the present study, increased levels of miR-15a-5p and pro-inflammatory cytokines in RAW264.7 macrophages stimulated with LPS were observed. It was subsequently confirmed that TNIP2 is a direct target of miR-15a-5p. Furthermore, miR-15a-5p inhibitor was found to protect against LPS-induced pro-inflammatory cytokine secretions by inhibiting NF- $\kappa \mathrm{B}$ signal activation in macrophages. In an in vivo model, miR-15a-5p inhibitor exhibited anti-inflammatory and organ-protective effects. In particular, the effects of miR-15a-5p inhibitor were significantly eliminated by TNIP2-small interfering RNA (siRNA) treatment.

In summary, the present study provided novel evidence that miR-15a-5p is a vital regulator and is involved in the inflammatory process in sepsis. miR-15a-5p may regulate inflammatory responses by inhibiting TNIP2, while activating the $\mathrm{NF}-\kappa \mathrm{B}$ pathway.

\section{Materials and methods}

Animals. Healthy adult male C57BL/6 mice (age, 6-8 weeks; weight, 20-22 g) were purchased from the Experimental Animal Center of the Fourth Military Medical University. A total of 50 C57BL/6 mice were used in the present investigation. All animals were housed under standard conditions at room temperature $\left(22-24^{\circ} \mathrm{C}\right)$ and humidity (60-65\%) on a 12-h light/dark cycle with ad libitum supply of food and water. This study was performed in accordance to the National Institutes of Health Guide for the Care and Use of Laboratory Animals (29) and was approved by the Committee of Experimental Animals of the Affiliated Hospital of Medical School of Ningbo University. The mice were acclimated to the environment for 1 week prior to experiments.
Animal model. C57BL/6 mice were intraperitoneally injected with LPS (10 mg/kg; Sigma-Aldrich; Merck KGaA) to induce sepsis. For treatment, the inhibitor control (80 mg/kg/day; 5'-CAGUACUUUUGUGUAGUACAA-3'; Shanghai GenePharma Co., Ltd.), miR-15a-5p inhibitor (80 mg/kg/day; Guangzhou RiboBio Co., Ltd.) or miR-15a-5p inhibitor $(80 \mathrm{mg} / \mathrm{kg} / \mathrm{day}$; 5'-CACUGGUACAAGGGUUGG GAGA-3'; Shanghai GenePharma Co., Ltd.) + TNIP2-siRNA ( $80 \mathrm{mg} / \mathrm{kg} /$ day) were injected by caudal vein for 3 consecutive days as previously described (30), followed by LPS (10 mg/kg) injection at $24 \mathrm{~h}$ following the last administration. The mice were anaesthetized with sodium pentobarbital $(30 \mathrm{mg} / \mathrm{kg}$; intraperitoneal injection) and sacrificed by cervical dislocation. The blood was subsequently collected for further analysis at $24 \mathrm{~h}$ after LPS injection. The duration of the experiment was 4 days in total, and the health and behavior of all mice were monitored every 2 days. No mice died during the experiment. The experiment was terminated when the mice lost $>15 \%$ of their body weight, and throughout the experiment attempts were made to alleviate the pain of the mice.

ELISA and biochemical marker detection. At $24 \mathrm{~h}$ after LPS injection, blood was collected from each mouse. The blood was centrifuged for $15 \mathrm{~min}$ at $1,600 \mathrm{x} \mathrm{g}$ at $4^{\circ} \mathrm{C}$ and the serum was collected for biochemical analysis. The levels of cytokines, including IL-1 $\beta$ (cat. no. PI301), IL-6 (cat. no. PI326) and TNF- $\alpha$ (cat. no. PT512), were examined using ELISA (Beyotime Institute of Biotechnology). The signal produced from each well was detected at a wavelength of $450 \mathrm{~nm}$. Alanine aminotransferase (ALT), aspartate aminotransferase (AST), creatine $(\mathrm{Cr})$ and blood urea nitrogen (BUN; all kits supplied by Nanjing Jiancheng Bioengineering Institute) were detected in the serum to evaluate organ function, according to the manufacturer's protocols.

Cell culture and LPS treatment. The mouse macrophage cell line RAW264.7 (cat. no. (ATCC ${ }^{\circledR}$ TIB-71 ${ }^{\mathrm{TM}}$; American Type Culture Collection) and 293 cells (cat. no. (ATCC ${ }^{\circledR}$ CRL-1573; American Type Culture Collection) were grown in DMEM (Gibco; Thermo Fisher Scientific, Inc.), including 10\% FBS (Thermo Fisher Scientific, Inc.) and 1\% penicillin and streptomycin (Beyotime Institute of Biotechnology). The cells were maintained at $37^{\circ} \mathrm{C}$ with $5 \% \mathrm{CO}_{2}$. In the present study, a concentration of $1 \mu \mathrm{g} / \mathrm{ml}$ LPS was used to stimulate RAW264.7 cells for $4 \mathrm{~h}$, and cells were harvested for further experiments.

Cell transfection and reagents. Prior to transfection, cells were changed to antibiotic-free media for $24 \mathrm{~h}$, then RAW264.7 macrophage cells $\left(5 \times 10^{4}\right.$ cells per well) were transfected with $0.2 \mu \mathrm{M}$ control-siRNA (cat. no. sc-36869; Santa Cruz Biotechnology, Inc.), $0.2 \mu \mathrm{M}$ TINP2-siRNA (cat. no. sc-44638; Santa Cruz Biotechnology, Inc.), 100 nM inhibitor control (Shanghai GenePharma Co., Ltd.; 5'-CAGUACUUUUGU GUAGUACAA-3'), 100 nM miR-15a-5p inhibitor (Shanghai GenePharmaCo.,Ltd.; 5'-CACUGGUACAAGGGUUGGGAG A-3') or $100 \mathrm{nM}$ miR-15a-5p inhibitor + 0.2 $\mu \mathrm{M}$ TNIP2-siRNA using Lipofectamine ${ }^{\circledR} 2000$ reagent (Invitrogen; Thermo Fisher Scientific, Inc.), according to the manufacturer's protocol. The efficiency of cell transfection was measured using reverse 
transcription quantitative-PCR (RT-qPCR) and western blot assays with samples collected $48 \mathrm{~h}$ after cell transfection. Cells without any treatment were used as the control.

Detection of IL-1 $\beta, I L-6$ and TNF- $\alpha$ level in cell supernatant. RAW264.7 cells were transfected with inhibitor control, miR-15a-5p inhibitor, or miR-15a-5p inhibitor+TNIP2-siRNA for $48 \mathrm{~h}$. Then the cells were subjected to $1 \mu \mathrm{g} / \mathrm{ml}$ LPS for $4 \mathrm{~h}$. Subsequently, the expression levels of IL-1 $\beta$, IL- 6 and TNF- $\alpha$ in the cell supernatant were also detected using ELISA kits, according to the manufacturer's protocols.

Dual-luciferase reporter assay. Target prediction database (TargetScan 7.2; http://www.targetscan.org/vert_72/) was used to examine the association between miR-15a-5p and TNIP2 . The results indicated that TNIP2 was a potential target of miR-15a-5p. The 3'-UTR of TNIP2, containing wild-type (WT) or mutant (MUT) target sites for miR-15a-5p, was subsequently amplified and inserted into the pGL3-Control Vector (Promega Corporation) to form the reporter vector TNIP2-WT or TNIP2-MUT, respectively. For the luciferase reporter assay, the luciferase reporter vectors and $50 \mathrm{nM}$ mimic control (Shanghai GenePharma Co., Ltd.; sense, 5'-UUCUCCGAA CGUGUCACGUTT-3'; and antisense, 5'-ACGUGACACGUU CGGAGAATT-3') or $50 \mathrm{nM}$ miR-15a-5p mimic (Shanghai GenePharma Co., Ltd.; sense, 5'-UCUCCCAACCCUUGU ACCAGUG-3'; and antisense, 5'-CUGGUACAAGGGUUG GGAGAUU-3') were co-transfected into 293 cells $\left(5 \times 10^{4}\right.$ cells per well) using Lipofectamine 2000 for $48 \mathrm{~h}$ according to the manufacturer's protocol. Dual-Luciferase Reporter assay system (Promega Corporation) was used to measure the luciferase activity and results were normalized to that of Renilla luciferase, according to the manufacturer's protocol. Each experiment was repeated in triplicate.

$R T-q P C R$. Total RNA was extracted from serum samples and RAW264.7 macrophages using TRIzol reagent (Invitrogen; Thermo Fisher Scientific, Inc.), according to the manufacturer's protocol. In order to detect mRNA expression levels, PrimeScript ${ }^{\mathrm{TM}}$ RT reagent kit (Takara Biotechnology Co., Ltd.) was used for cDNA synthesis. The reaction conditions were as follows: Initial annealing at $25^{\circ} \mathrm{C}$ for $5 \mathrm{~min}$, followed by extension at $42^{\circ} \mathrm{C}$ for $60 \mathrm{~min}$ and termination at $80^{\circ} \mathrm{C}$ for $2 \mathrm{~min}$. The expression levels of miR-15a-5p and TNIP2 were measured using Real-time PCR system (Applied Biosystems; Thermo Fisher Scientific, Inc.) with the SYBR Green PCR kit (Takara Biotechnology Co., Ltd.), according to the manufacturer's protocols. GAPDH or U6 was used as an internal control. Primer sequences (all provided by Sangon Biotech Co., Ltd.) are as following: U6 forward, 5'GCTTCGGCAGCACATATACTAAAAT3' and reverse, 5'CGCTTCACGAATTTGCGTGTCAT3'; GAPDH forward, 5'CTTTGGTATCGTGGAAGGACTC3' and reverse, 5'GTAGAGGCAGGGATGATGTTCT3'; miR-15a-5p forward, 5'GGGTAGCAGCACATAATGGTT TGTG3' and reverse, 5'CAGTGCGTGTCGTGGAGT3'; TNIP2 forward, 5'-CTAAAGAGGCGGCAGGTCCCTC-3' and reverse, 5'-CAAGATGACCTTCCAGTGAC-3'. The following thermocycling conditions were used: 35 cycles of initial denaturation at $95^{\circ} \mathrm{C}$ for $15 \mathrm{sec}$, annealing at $60^{\circ} \mathrm{C}$ for $1 \mathrm{~min}$, extension at $72^{\circ} \mathrm{C}$ for $1 \mathrm{~min}$; and a final extension step at $72^{\circ} \mathrm{C}$ for $10 \mathrm{~min}$. The relative expression of genes was determined using the $2^{-\Delta \Delta \mathrm{Cq}}$ method (31).

Western blot assay. Total protein was obtained from cells using RIPA lysis buffer (Beyotime Institute of Biotechnology). Protein concentration was measured using a bicinchoninic acid protein assay kit (Thermo Fisher Scientific, Inc.). Equal amounts of protein $(40 \mu \mathrm{g})$ were separated by $10 \%$ SDS-PAGE (Bio-Rad Laboratories, Inc.) and transferred onto PVDF membranes (EMD Millipore). Membranes were subsequently blocked with 5\% fat-free skim milk in PBS-Tween (PBST), containing $0.05 \%$ Tween-20, for $1 \mathrm{~h}$ at room temperature, and incubated with the following primary antibodies at $4^{\circ} \mathrm{C}$ overnight: TNIP2 (1:1,000; cat. no. ab205925; Abcam), p-p65 (1:1,000; cat. no. ab86299; Abcam), p65 (1:1,000; cat. no. ab16502; Abcam) and $\beta$-actin (1:2,000; cat. no. ab8227; Abcam). The membranes were washed in triplicate with PBST and then incubated with horseradish peroxidase-conjugated goat anti-rabbit IgG H\&L (1:2,000; cat. no. Ab7090; Abcam) at room temperature for $1 \mathrm{~h}$. The protein bands were visualized using the ECL detection reagent (Beyotime Institute of Biotechnology). The intensity of each band was quantified using Image Lab $^{\text {TM }}$ Software (version 5.2.1; Bio-Rad Laboratories Inc.).

Statistical analysis. SPSS 21.0 statistical software (IBM Corp.) was used to analyze data. All experiments were performed at least three times. Measurement data were presented as the mean \pm standard deviation. Differences between multiple groups were tested using ANOVA followed by Student-Newman-Keuls tests. Differences between two groups were analyzed by Student's t-test. $\mathrm{P}<0.05$ was considered to indicate a statistically significant difference.

\section{Results}

miR-15a-5p and inflammatory factors' expression levels are significantly increased in RAW264.7 macrophages after LPS treatment. After RAW264.7 macrophages were stimulated with LPS for $4 \mathrm{~h}$, the expression levels of miR-15a-5p and inflammatory factors were detected by RT-qPCR and ELISAs, respectively. IL-1 $\beta$, IL- 6 and TNF- $\alpha$ levels were detected to evaluate the inflammatory response. The results indicated that the expression levels of IL-1 $\beta$, IL- 6 and TNF- $\alpha$ were significantly increased in RAW264.7 macrophages after treatment with LPS compared with the control group (Fig. 1A-C). Additionally, the data showed that the expression level of miR-15a-5p was increased with LPS stimulation (Fig. 1D).

TNIP2 is a target gene of miR-15a-5p. In order to investigate the molecular mechanism of action for miR-15a-5p, the present study predicted the potential targets of miR-15a-5p using a bioinformatics prediction tool (TargetScan). The binding sites between miR-15a-5p and TNIP2 were observed and are presented in Fig. 2A. A dual-luciferase reporter assay was subsequently used to further verify that there is a specific regulatory connection between miR-15a-5p and TNIP2. The results showed that increased expression levels of miR-15a-5p decreased the luciferase activity of WT 3'-UTR segment of 

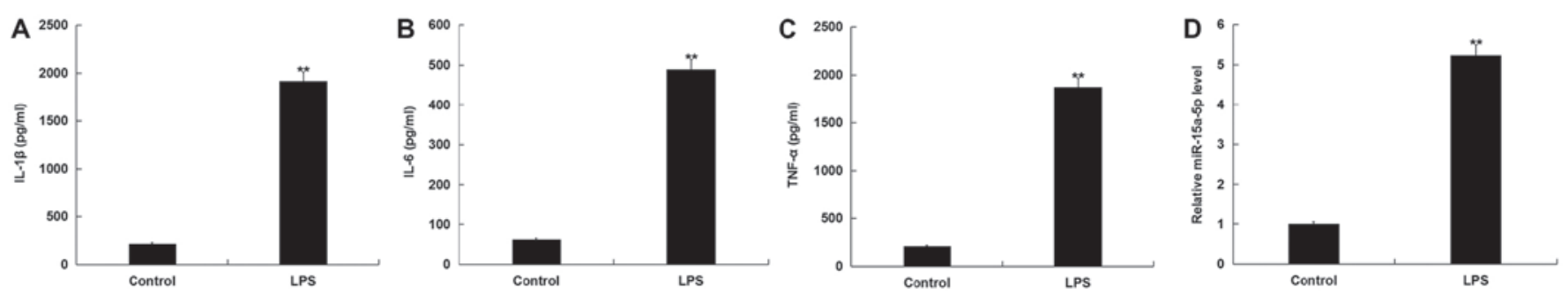

Figure 1. Expression levels of miR-15a-5p and inflammatory factors are significantly upregulated in RAW264.7 macrophages treated with LPS. RAW264.7 macrophages were stimulated with $1 \mu \mathrm{g} / \mathrm{ml}$ LPS for $4 \mathrm{~h}$ and the expression levels of (A) IL-1 $\beta$, (B) IL-6, and (C) TNF- $\alpha$ were examined using ELISAs. (D) Detection of miR-15a-5p expression levels in RAW264.7 macrophages. ${ }^{* *} \mathrm{P}<0.01$ vs. control. IL, interleukin; LPS, lipopolysaccharide; miR, microRNA; TNF- $\alpha$, tumor necrosis factor- $\alpha$.
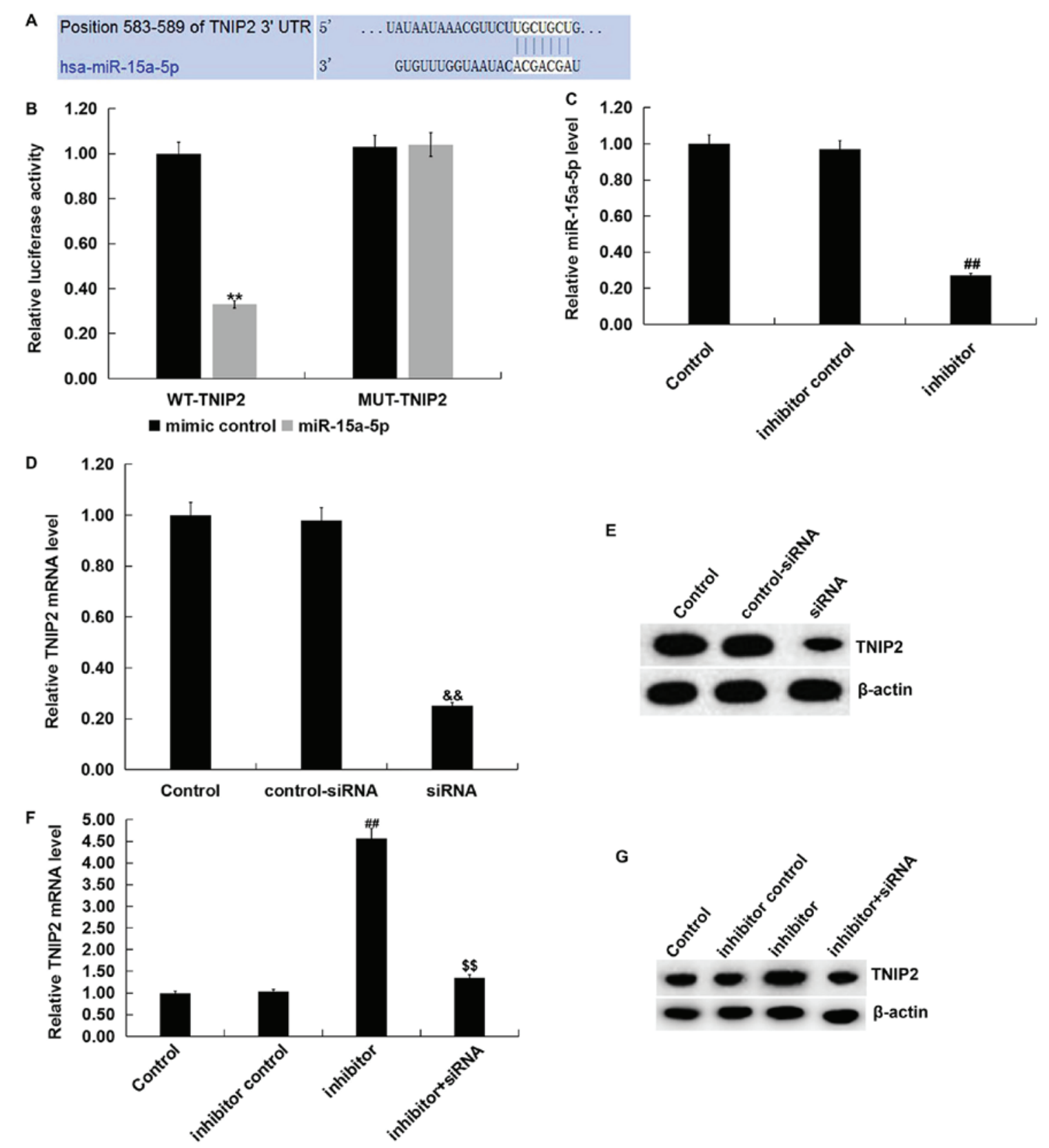

G

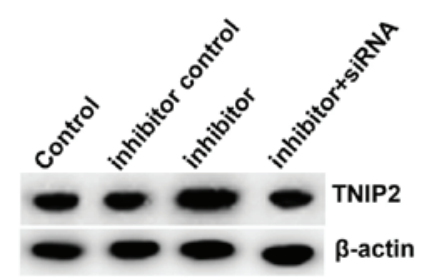

Figure 2. TNIP2 is a direct target of miR-15a-5p. (A) The binding sites between miR-15a-5p and TNIP2 3'-UTR. (B) Dual-luciferase reporter assays were performed to measure the luciferase activities. ${ }^{* *} \mathrm{P}<0.01$ vs. mimic control. (C) Inhibitor control or miR-15a-5p inhibitor was transfected into RAW264.7 macrophages for $48 \mathrm{~h}$ to detect the mRNA levels of miR-15a-5p. Control-siRNA or TNIP2-siRNA was transfected into RAW264.7 macrophages for $48 \mathrm{~h}$ to detect the TNIP2 (D) mRNA and (E) protein levels. Following transfection with miR-15a-5p inhibitor, inhibitor control, or miR-15a-5p inhibitor + TNIP2-siRNA, the (F) mRNA and (G) protein expression level of TNIP2 in RAW264.7 macrophages was measured. ${ }^{\# /} \mathrm{P}<0.01$ vs. inhibitor control; ${ }^{\& \&} \mathrm{P}<0.01$ vs. control-siRNA; ${ }^{\$ \$} \mathrm{P}<0.01$ vs. inhibitor. miR, microRNA; MUT, mutant; LPS, lipopolysaccharide; siRNA, small interfering RNA; TNIP, tumor necrosis factor- $\alpha$ induced protein 3-interacting protein; WT, wild-type; UTR, untranslated region. 

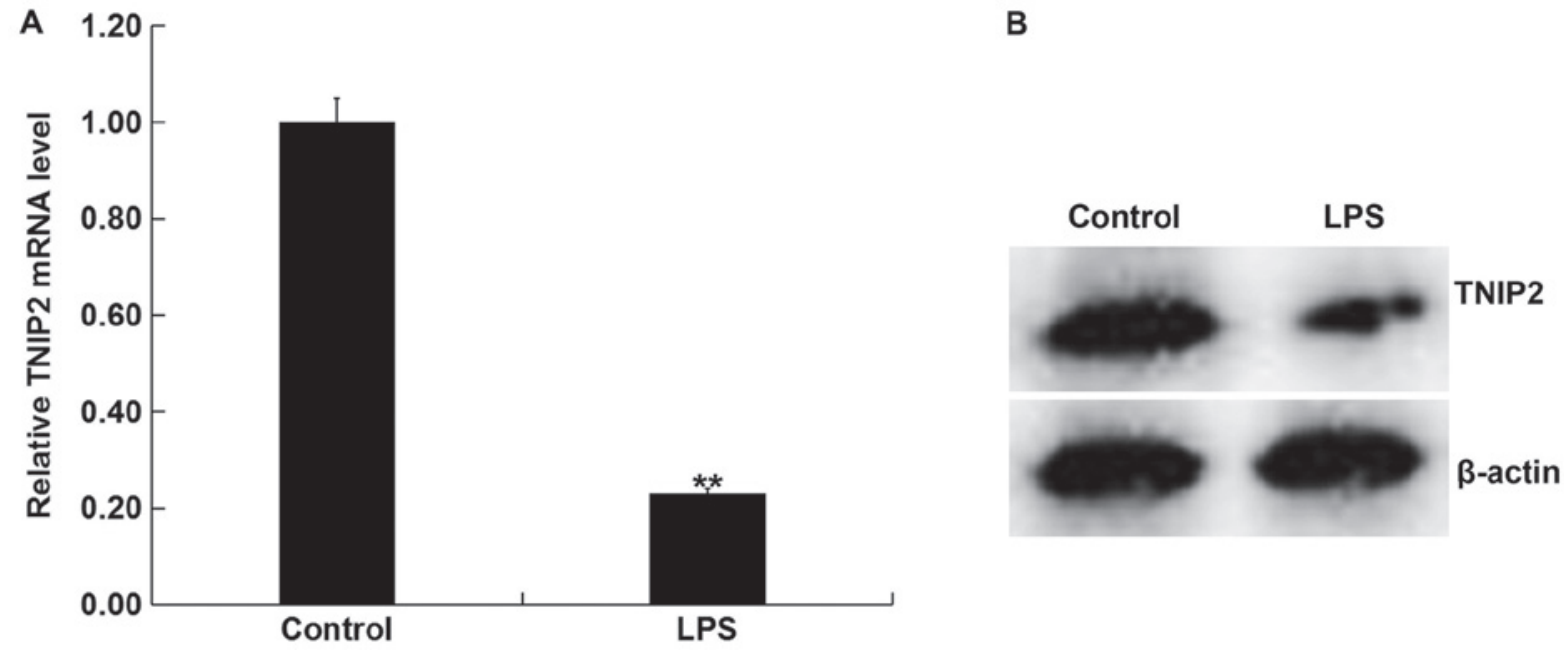

Figure 3. Expression of TNIP2 is suppressed in RAW264.7 macrophages following LPS treatment. (A) The mRNA expression levels of TNIP2 were reduced in RAW264.7 macrophages treated with LPS. (B) The protein levels of TNIP2 were detected by western blot analysis in RAW264.7 macrophages following LPS treatment. ${ }^{* *} \mathrm{P}<0.01$ vs. control. LPS, lipopolysaccharide; TNIP, tumor necrosis factor- $\alpha$ induced protein 3 -interacting protein.

TNIP2 compared with mimic control group, however no significant differences were observed in the luciferase activity of TNIP2-MUT (Fig. 2B). Together, these results suggested that TNIP2 was a direct target of miR-15a-5p.

Furthermore, the present study examined whether miR-15a-5p could regulate the expression of TNIP2 in RAW264.7 macrophages. The inhibitor control, miR-15a-5p inhibitor, control-siRNA, TNIP2-siRNA or miR-15a-5p inhibitor + TNIP2-siRNA were transfected into RAW264.7 macrophages for $48 \mathrm{~h}$. As indicated in Fig. 2C, it was observed that compared with the inhibitor control group, the expression levels of miR-15a-5p were significantly suppressed in RAW264.7 macrophages transfected with the miR-15a-5p inhibitor. Meanwhile, compared with the control-siRNA group reduced mRNA and protein expression levels of TNIP2 were detected when RAW264.7 macrophages were transfection with TNIP2-siRNA (Fig. 2D and E). Additionally, RT-qPCR and western blot analyses demonstrated that the mRNA and protein expression levels of TNIP2 were significantly higher in RAW264.7 macrophages after miR-15a-5p inhibitor transfection compared with the inhibitor control group (Fig. 2F and G). This increase was reversed by TNIP2-siRNA co-transfection. These data indicated that TNIP2 was a direct target of miR-15a-5p. Furthermore, a negative association between miR-15a-5p and TNIP2 was shown, which may be related to the progression of sepsis.

Expression of TNIP2 is reduced in LPS-activated RAW264.7 macrophages. In order to further verify the difference in expression levels of TNIP2 between the LPS-activated RAW264.7 macrophages and untreated RAW264.7 macrophages, RAW264.7 macrophages were treated with LPS for $4 \mathrm{~h}$. It was indicated that TNIP2 mRNA levels were significantly decreased in the LPS treatment group compared with the control group (Fig. 3A). Consistently, the protein expression of TNIP2 appeared reduced in RAW264.7 macrophages upon LPS activation (Fig. 3B). These data suggested that LPS may inhibit the expression of TNIP2 in RAW264.7 macrophages.
miR-15a-5p inhibitor suppresses LPS-induced inflammatory factors' expression in RAW264.7 macrophages by TNIP2 regulation. The expression levels of IL-1 $\beta$, IL-6 and TNF- $\alpha$ following transfection and LPS treatment were detected. Results from the ELISAs showed that LPS triggered an increased secretion of IL- $1 \beta$, IL- 6 and TNF- $\alpha$ from RAW264.7 macrophages compared with the control group. Additionally, inhibition of miR-15a-5p suppressed the secretion of IL-1 $\beta$, IL- 6 and TNF- $\alpha$ in RAW264.7 macrophages which were stimulated by LPS, compared with the LPS + inhibitor control treatment group. Meanwhile, the effects were reversed by TNIP2-siRNA, and the protein expression levels of IL-1 $\beta$, IL- 6 and TNF- $\alpha$ were increased in the TNIP2-siRNA co-transfection group compared with the inhibitor group (Fig. 4A-C).

miR-15a-5p inhibitor significantly affects the activity of $N F-\kappa B$ in LPS-induced RAW264.7 macrophages via TNIP2 regulation. The effect of miR-15a-5p inhibitor on LPS-induced $\mathrm{NF}-\kappa \mathrm{B}$ activation was subsequently examined. After transfecting RAW264.7 macrophages with miR-15a-5p inhibitor or miR-15a-5p inhibitor + TNIP2-siRNA for $48 \mathrm{~h}$, LPS was used to stimulate the cells for $4 \mathrm{~h}$. As indicated in Fig. 5, the protein expression levels of p-p65 were enhanced in the LPS treatment group compared with the control group (Fig. 5A and B). Compared with the LPS + inhibitor control treatment group, miR-15a-5p inhibitor significantly inhibited the expression of p-p65 in LPS-treated cells, whereas TNIP2 downregulation reversed these effects. These data suggested that miRNA-15a-5p was involved in inflammatory progression by regulating the activation of the $N F-\kappa B$ pathway and by targeting TNIP2 in vitro.

Depletion of TNIP2 eliminates the effects of miR-15a-5p inhibitor in LPS-induced septic mice. To assess whether miRNA-15a-5p was involved in the inflammatory progression in septic mice, a mouse model of sepsis was established using LPS followed by treatment with miR-15a-5p inhibitor or miR-15a-5p inhibitor + TNIP2-siRNA. It was confirmed 

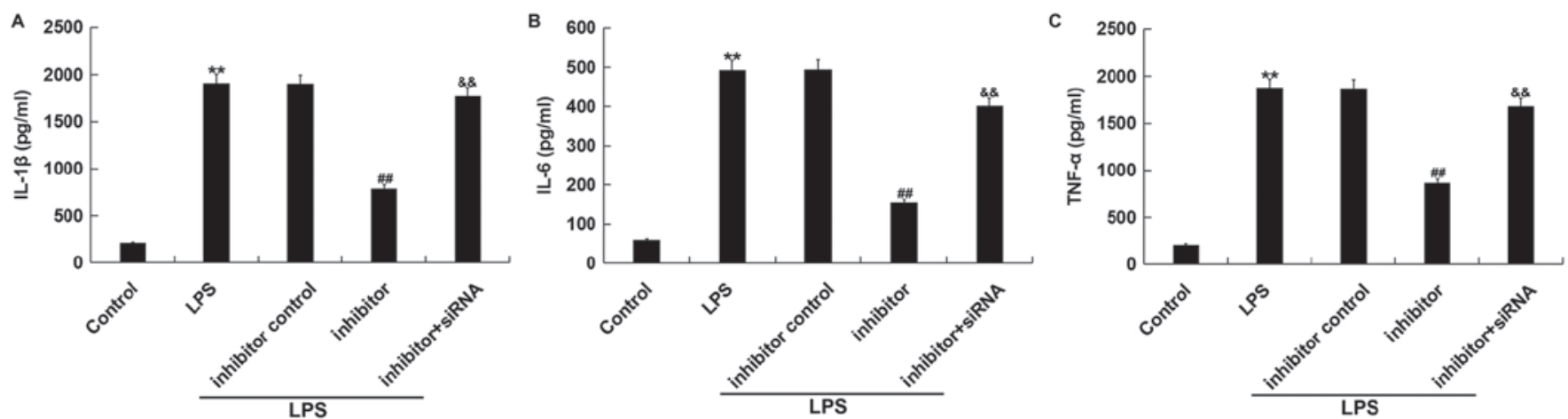

Figure 4. miR-15a-5p downregulation suppresses the LPS-induced inflammatory factors released in RAW264.7 macrophages. RAW264.7 macrophage cells were transfected with or without inhibitor control, miR-15a-5p inhibitor or miR-15a-5p inhibitor + TNIP2-siRNA for $48 \mathrm{~h}$, and cells were subsequently stimulated with $1 \mu \mathrm{g} / \mathrm{ml}$ LPS for $4 \mathrm{~h}$. The expression levels of inflammatory factors (A) IL-1 $\beta$, (B) IL-6 and (C) TNF- $\alpha$ in cells from the various treatment groups were detected using ELISAs. ${ }^{* *} \mathrm{P}<0.01$ vs. control; ${ }^{\# \#} \mathrm{P}<0.01$ vs. LPS+inhibitor control; ${ }^{\& \&} \mathrm{P}<0.01$ vs. LPS+inhibitor. LPS, lipopolysaccharide; miR, microRNA; IL, interleukin; siRNA, small interfering RNA; TNF, tumor necrosis; TNIP, TNF- $\alpha$ induced protein 3 -interacting protein.

A

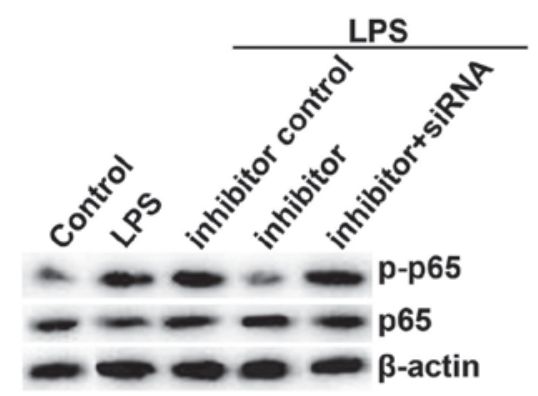

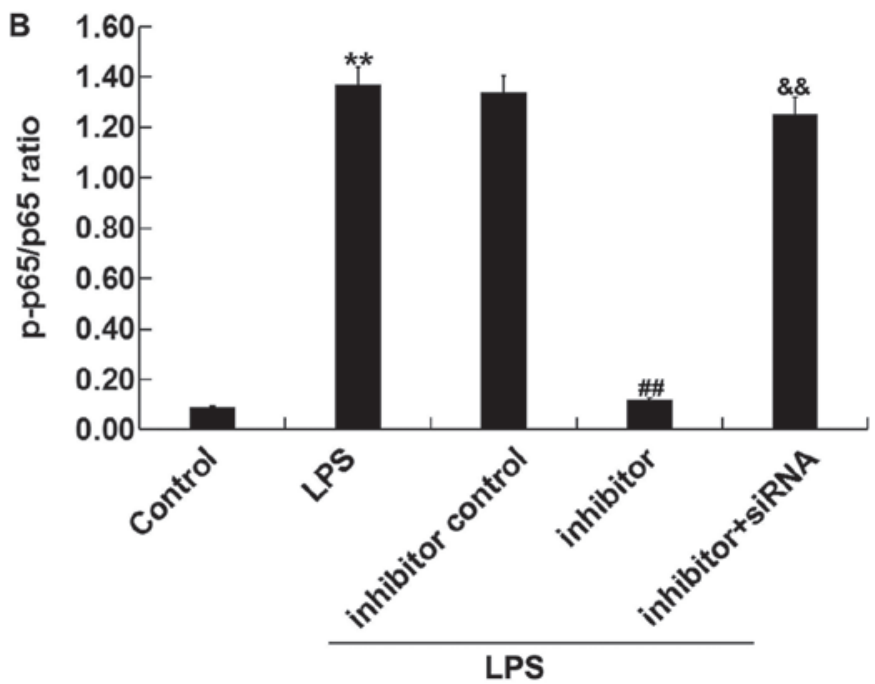

Figure 5. miR-15a-5p inhibitor inhibits LPS-induced NF-кB activation in RAW264.7 macrophages and TNIP2-siRNA reverses the effects. RAW264.7 macrophage cells were transfected with or without inhibitor control, miR-15a-5p inhibitor or miR-15a-5p inhibitor + TNIP2-siRNA for $48 \mathrm{~h}$, and cells were stimulated with $1 \mu \mathrm{g} / \mathrm{ml}$ LPS for $4 \mathrm{~h}$. (A) Protein expression level of p-p65 and p65 was detected using western blot assays. (B) The ratio of p-p65/p65. ${ }^{* * *} \mathrm{P}<0.01 \mathrm{vs.} \mathrm{control;}$ ${ }^{\# \#} \mathrm{P}<0.01$ vs. LPS+inhibitor control; \&\& $\mathrm{P}<0.01$ vs. LPS+inhibitor. LPS, lipopolysaccharide; miR, microRNA; $p$, phosphorylated; siRNA, small interfering RNA; TNIP, tumor necrosis factor- $\alpha$ induced protein 3-interacting protein.

that compared with the inhibitor control injection group, miR-15a-5p inhibitor significantly decreased the level of miR-15a-5p in the serum of mice (Fig. 6A). TNIP2-siRNA significantly decreased the mRNA level of TNIP2 in the serum of mice compared to the control-siRNA group (Fig. 6B). ELISAs were carried out to detect the levels of IL-1 $\beta$, IL-6 and TNF- $\alpha$ in the serum of mice from the various treatment groups (LPS; LPS + inhibitor control; LPS + inhibitor; LPS + inhibitor + siRNA). As indicated in Fig. 7A-C, miR-15a-5p inhibitor significantly decreased the secretion of inflammatory factors, including IL-1 $\beta$, IL- 6 and TNF- $\alpha$, in the serum of LPS-treated mice. Additionally, compared with the LPS treatment alone group, the levels of kidney and liver injury markers, including Cr, BUN, ALT and AST, in the serum of LPS-treated mice were reduced in the miR-15a-5p inhibitor-administered group (Fig. 7D-G). The aforementioned effects of miR-15a-5p inhibitor on LPS-treated mice were eliminated by the TNIP2-siRNA combination therapy. These results indicated that miRNA-15a-5p promoted the inflammatory response by negatively regulating TNIP2 expression in septic mice.

\section{Discussion}

Previous studies have reported that sepsis is associated with an excessive inflammatory response $(1,20)$. A number of studies have shown that miRNAs participate in the sepsis-induced inflammatory response by affecting vital signaling elements. For example, Chen et al (32) found that miR-212-3p depressed LPS-induced inflammatory responses by targeting high-mobility group protein B in sepsis. Additionally, Wang et al (33) showed that upregulation of miR-130b suppressed severe lung inflammation in the sepsis mouse model by LPS stimulation. However, the underlying mechanisms of action for miR-15a-5p participation in the progression of sepsis remain unknown. Based on the aforementioned literature, the aim of the present study was to elucidate the effect of 
A

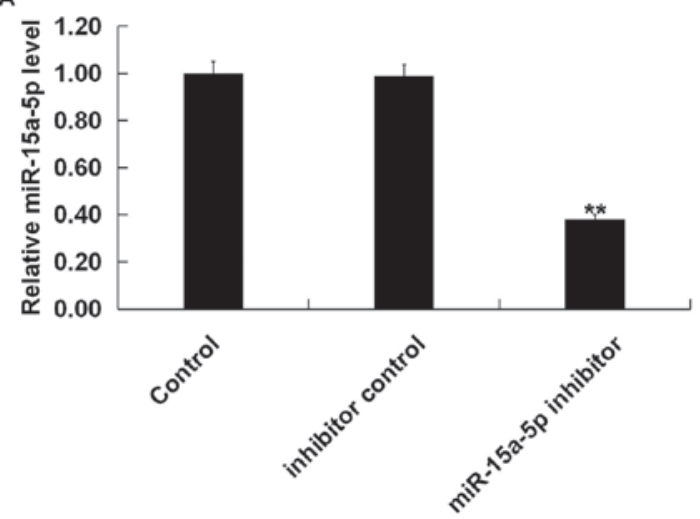

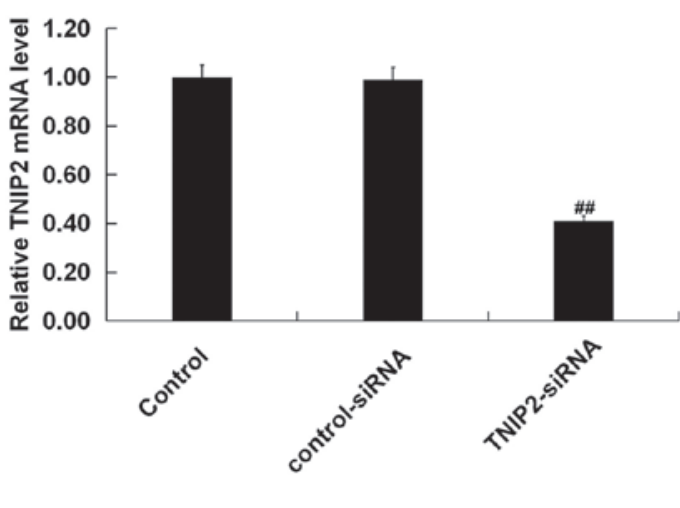

Figure 6. Effect of miR-15a-5p inhibitor and TNIP2-siRNA on miR-15a-5p and TNIP2 expression in mice. (A) The level of miR-15a-5p in the serum of mice was detected using RT-qPCR. (B) The mRNA level of TNIP2 in the serum of mice was detected using RT-qPCR. "*P<0.01 vs. inhibitor control; ${ }^{\# /} \mathrm{P}<0.01$ vs. control-siRNA. miR, microRNA; siRNA, small interfering RNA; RT-qPCR, reverse transcription-quantitative PCR.
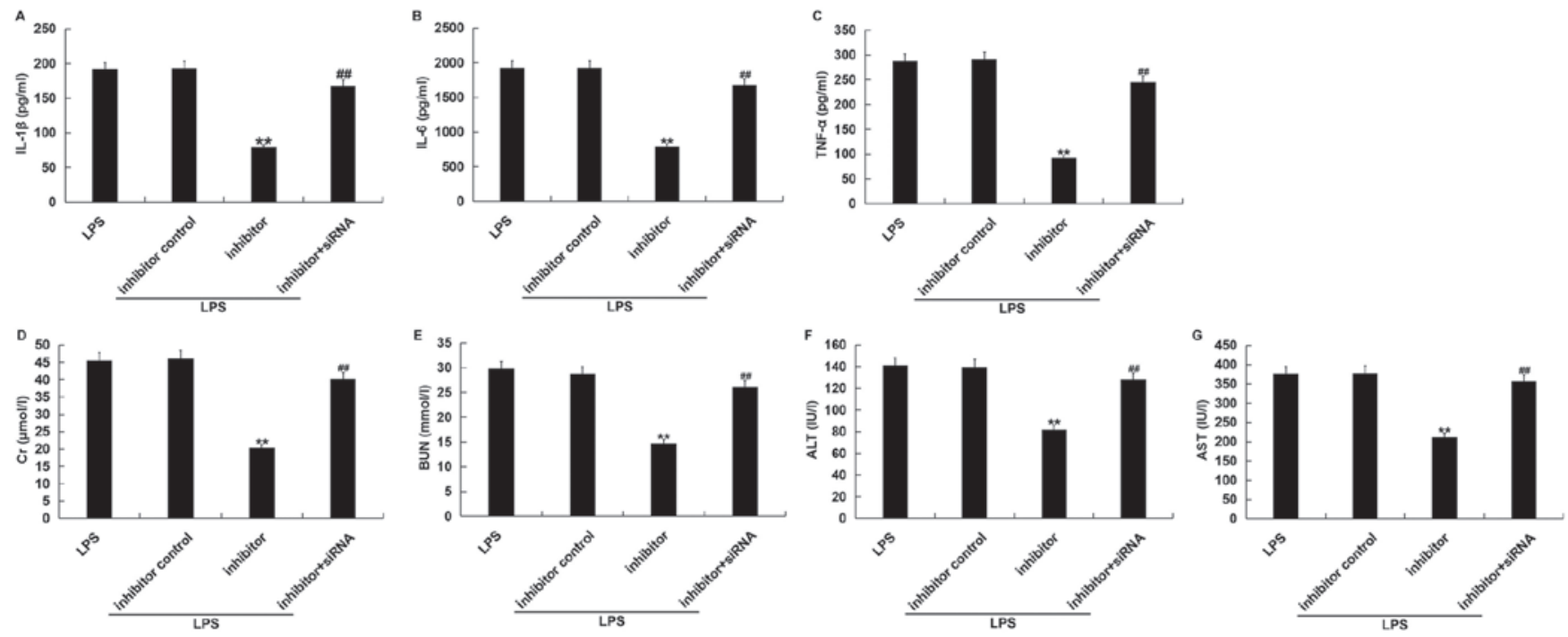

Figure 7. TNIP2-siRNA eliminates the effects of miR-15a-5p inhibitor in LPS-induced septic mice. The serum (A) IL-1 $\beta$, (B) IL-6 and (C) TNF- $\alpha$ levels were evaluated using ELISAs. The serum (D) Cr, (E) BUN, (F) ALT and (G) AST levels were evaluated in the various treatment groups. "** $<<0.01$ vs. LPS+inhibitor control; ${ }^{\# \prime} \mathrm{P}<0.01$ vs. LPS+inhibitor. ALT, alanine aminotransferase; AST, aspartate aminotransferase; BUN, blood urea nitrogen; Cr, creatine; LPS, lipopolysaccharide; miR, microRNA; IL, interleukin; siRNA, small interfering RNA; TNF, tumor necrosis factor; TNIP, TNF- $\alpha$ induced protein 3-interacting protein.

miR-15a-5p in the sepsis inflammatory response. The present study provided evidence that miR-15a-5p inhibitor suppressed the inflammatory response in both cultured macrophages and in a septic mouse model by repressing the activation of the $\mathrm{NF}-\kappa \mathrm{B}$ signaling pathway and by targeting TNIP2.

RT-qPCR assays and ELISAs were used to detect miR-15a-5p expression levels as well as inflammatory factors, including IL-1 $\beta$, IL- 6 and TNF- $\alpha$, in LPS-induced RAW264.7 macrophages. In accordance with the present study, previous studies have also reported that IL-1 $\beta$, IL- 6 and TNF- $\alpha$ expression was enhanced in LPS-treated RAW264.7 macrophages $(34,35)$. The present results also found that miR-15a-5p was significantly upregulated in LPS-treated RAW264.7 macrophages. Therefore, inhibiting miR-15a-5p expression may block the progression of inflammation and serve an anti-inflammatory role in sepsis. Additionally, TNIP2 was confirmed as a direct target of miR-15a-5p through the dual-luciferase reporter assay. Therefore, revealing a potential mechanism of action for TNIP2 with the inflammatory response is crucial. In order to confirm the hypothesis, TNIP2-knockdown was performed using siRNA. The roles of miR-15a-5p inhibitor and TNIP2-siRNA were investigated in RAW264.7 macrophages with LPS stimulation. It was observed that miRNA-15a-5p inhibitor negatively regulated TNIP2 expression in RAW264.7 macrophages and that the expression levels of TNIP2 in RAW264.7 macrophages with LPS treatment, significantly decreased. Taking all of the aforementioned data into consideration, this study suggests that TNIP 2 is a direct target gene of miRNA-15a-5p and a negative relationship between miR-15a-5p and TNIP2 is indicated. This relationship may regulate the progression of sepsis.

TNIP2, the binding partner of zinc finger protein A20 (A20), was first discovered in a yeast two-hybrid screen. TNIP2 was found to regulate $\mathrm{NF}-\kappa \mathrm{B}$ by binding to A20, a well-known anti-inflammatory signaling molecule (36). Overexpression of TNIP2 has been reported to inhibit NF- $\mathrm{B}$ activation and result in 
cell proliferation in human cancer types (37). Therefore, the effects of miR-15a-5p inhibitor or miR-15a-5p inhibitor + TNIP2-siRNA on inflammatory factor (TNF- $\alpha$, IL-1 $\beta$ and IL-6) expression levels and the NF- $\kappa$ B signal pathway in LPS stimulated RAW264.7 macrophages were further investigated. The results demonstrated that miR-15a-5p inhibitor alleviated inflammatory responses in macrophages induced by LPS stimulation, as indicated by decreased levels of IL-1 $\beta$, IL- 6 and TNF- $\alpha$. However, there is some disagreement on whether IL-1 $\beta$ is expressed in RAW264.7 macrophages. For example, Pelegrin et al (38) reported that RAW264.7 macrophages do not release mature IL-1 $\beta$. However, a number of other studies have reported that RAW264.7 macrophages secrete mature IL-1 $\beta$, which is consistent with the results of the current study (39-41). Furthermore, it was found that the activation of the NF- $\mathrm{NB}$ signaling was inhibited by miR-15a-5p inhibitor in LPS-induced macrophages compared with the control group, indicated by reduced p-p65 expression and increased TINP2 expression level. However, all of the observed effects of the miR-15a-5p inhibitor on LPS-stimulated macrophages were counteracted by TNIP2-siRNA. These data revealed that miRNA-15a-5p was involved in the inflammatory progression in macrophages through modulating TNIP2.

In the current study a mouse model was also established by injecting LPS $(10 \mathrm{mg} / \mathrm{kg})$ to induce sepsis. The results revealed that the levels of inflammatory factors (IL-1 $\beta$, IL- 6 and TNF- $\alpha$ ) and organ damage markers ( $\mathrm{Cr}, \mathrm{BUN}, \mathrm{ALT}$ and AST) were decreased in the miR-15a-5p inhibitor + LPS group compared with the LPS group, while knockdown of TNIP2 reversed these effects. These results indicated that miRNA-15a-5p may promote the development of inflammation by negatively regulating TNIP2 expression in septic mice.

Overall, the present study demonstrated the anti-inflammatory activity of miRNA-15a-5p inhibitor in both in vitro and in vivo inflammatory models. To the best of our knowledge, this study is the first to examine the mechanisms of action for miRNA-15a-5p in the anti-inflammatory field. The results of the present study confirmed that miR-15a-5p inhibitor prevented the activation of the $\mathrm{NF}-\kappa \mathrm{B}$ signaling pathway by negatively regulating TNIP2 expression, suppressing the inflammatory response and therefore providing novel insights into the treatment of sepsis. However, this study is only a preliminary analysis of the role of miRNA-15a-5p in sepsis and further investigation of its role is required. For example, the role of TNIP2 alone in sepsis should be studied. Additionally, $\mathrm{NF}-\kappa \mathrm{B}$ activity, as well as the inflammatory factor levels in RAW264.7 cells with upregulated/downregulated TNIP2 expression upon LPS treatment should be investigated.

\section{Acknowledgements}

Not applicable.

\section{Funding}

No funding was received.

\section{Availability of data and materials}

The datasets used and/or analyzed during the current study are available from the corresponding author on reasonable request.

\section{Authors' contributions}

YL designed the current study, collected and analyzed the data, performed statistical analysis, and prepared the manuscript. $\mathrm{ZH}$ collected and analyzed the data, performed statistical analysis, and prepared the manuscript.

\section{Ethics approval and consent to participate}

The current study was performed in accordance with the National Institutes of Health Guide for the Care and Use of Laboratory Animals and was approved by the Committee of Experimental Animals of the Affiliated Hospital of Medical School of Ningbo University.

\section{Patient consent for publication}

Not applicable.

\section{Competing interests}

The authors declare that they have no competing interests.

\section{References}

1. Panagiotou A, Gaiao S and Cruz DN: Extracorporeal therapies in sepsis. J Intensive Care Med 28: 281-295, 2013.

2. Shane AL and Stoll BJ: Neonatal sepsis: Progress towards improved outcomes. J Infect 68 (Suppl 1): S24-S32, 2014.

3. Aziz M, Jacob A, Yang WL, Matsuda A and Wang P: Current trends in inflammatory and immunomodulatory mediators in sepsis. J Leukoc Biol 93: 329-342, 2013.

4. Bosmann M and Ward PA: The inflammatory response in sepsis. Trends Immunol 34: 129-136, 2013.

5. Burnham JP, Lane MA and Kollef MH: Impact of sepsis classification and multidrug-resistance status on outcome among patients treated with appropriate therapy. Crit Care Med 43: 1580-1586, 2015

6. Carchman EH, Rao J, Loughran PA, Rosengart MR and Zuckerbraun BS: Heme oxygenase-1-mediated autophagy protects against hepatocyte cell death and hepatic injury from infection/sepsis in mice. Hepatology 53: 2053-2062, 2011.

7. Hotchkiss RS, Moldawer LL, Opal SM, Reinhart K, Turnbull IR and Vincent JL: Sepsis and septic shock. Nat Rev Dis Primers 2: 16045, 2016.

8. Martin GS: Sepsis, severe sepsis and septic shock: Changes in incidence, pathogens and outcomes. Expert Rev Anti Infect Ther 10: 701-706, 2012.

9. Bartel DP: MicroRNAs: Target recognition and regulatory functions. Cell 136: 215-233, 2009.

10. Stefani G and Slack FJ: Small non-coding RNAs in animal development. Nat Rev Mol Cell Biol 9: 219-230, 2008

11. Nakajima TE, Yamada Y, Hamano T, Furuta K, Matsuda T, Fujita S, Kato K, Hamaguchi T and Shimada Y: Adipocytokines as new promising markers of colorectal tumors: Adiponectin for colorectal adenoma, and resistin and visfatin for colorectal cancer. Cancer Sci 101: 1286-1291, 2010.

12. Wichadakul D, Mhuantong W, Jongkaewwattana $A$ and Ingsriswang S: A computational tool for the design of live attenuated virus vaccine based on microRNA-mediated gene silencing. BMC Genomics 13 (Suppl 7): S15, 2012.

13. Cui XS, Sun SC, Kang YK and Kim NH: Involvement of microRNA-335-5p in cytoskeleton dynamics in mouse oocytes. Reprod Fertil Dev 25: 691-699, 2013.

14. Huang J, Sun Z, Yan W, Zhu Y, Lin Y, Chen J, Shen B and Wang J: Identification of microRNA as sepsis biomarker based on miRNAs regulatory network analysis. Biomed Res Int 2014: 594350, 2014

15. Srivastava M, Khurana P and Sugadev R: Lung cancer signature biomarkers: Tissue specific semantic similarity based clustering of digital differential display (DDD) data. BMC Res Notes 5: 617, 2012. 
16. Dalamaga M: Nicotinamide phosphoribosyl-transferase/visfatin: A missing link between overweight/obesity and postmenopausal breast cancer? Potential preventive and therapeutic perspectives and challenges. Med Hypotheses 79: 617-621, 2012.

17. Wang B, Hasan MK, Alvarado E, Yuan H, Wu H and Chen WY: NAMPT overexpression in prostate cancer and its contribution to tumor cell survival and stress response. Oncogene 30: 907-921, 2011.

18. Bi TQ, Che XM, Liao XH, Zhang DJ, Long HL, Li HJ and Zhao W: Overexpression of Nampt in gastric cancer and chemopotentiating effects of the Nampt inhibitor FK866 in combination with fluorouracil. Oncol Rep 26: 1251-1257, 2011.

19. Daniel P, Leśniowski B, Mokrowiecka A, Jasińska A, Pietruczuk $M$ and Małecka-Panas E: Circulating levels of visfatin, resistin and pro-inflammatory cytokine interleukin-8 in acute pancreatitis. Pancreatology 10: 477-482, 2010.

20. van der Poll T, van de Veerdonk FL, Scicluna BP and Netea MG: The immunopathology of sepsis and potential therapeutic targets. Nat Rev Immunol 17: 407-420, 2017.

21. Vachharajani VT, Liu T, Wang X, Hoth JJ, Yoza BK and McCall CE: Sirtuins link inflammation and metabolism. J Immunol Res 2016: 8167273, 2016.

22. Baldwin AJ: The NF-kappa B and I kappa B proteins: New discoveries and insights. Annu Rev Immunol 14: 649-683, 1996.

23. Wang ZM, Wan XH, Sang GY, Zhao JD, Zhu QY and Wang DM: miR-15a-5p suppresses endometrial cancer cell growth via Wnt/ $\beta$-catenin signaling pathway by inhibiting WNT3A. Eur Rev Med Pharmacol Sci 21: 4810-4818, 2017.

24. Long J, Jiang C, Liu B, Fang S and Kuang M: MicroRNA-15a-5p suppresses cancer proliferation and division in human hepatocellular carcinoma by targeting BDNF. Tumour Biol 37: 5821-5828, 2016.

25. Ergun S, Güney S, Temiz E, Petrovic N and Gunes S: Significance of miR-15a-5p and CNKSR3 as novel prognostic biomarkers in non-small cell lung cancer. Anticancer Agents Med Chem 18: 1695-1701, 2018.

26. Lee YJ, Choi DY, Choi IS, Kim KH, Kim YH, Kim HM, Lee K, Cho WG, Jung JK, Han SB, et al: Inhibitory effect of 4-O-methylhonokiol on lipopolysaccharide-induced neuroinflammation, amyloidogenesis and memory impairment via inhibition of nuclear factor-kappaB in vitro and in vivo models. J Neuroinflammation 9: 35, 2012.

27. Hayes JB, Sircy LM, Heusinkveld LE, Ding W, Leander RN, McClelland EE and Nelson DE: Modulation of macrophage inflammatory nuclear factor $\kappa \mathrm{B}(\mathrm{NF}-\kappa \mathrm{B})$ signaling by intracellular cryptococcus neoformans. J Biol Chem 291: 15614-15627, 2016.

28. Van Huffel S, Delaei F, Heyninck K, De Valck D and Beyaert R: Identification of a novel A20-binding inhibitor of nuclear factor-kappa B activation termed ABIN-2. J Biol Chem 276 30216-30223, 2001

29. Bayne K: Revised guide for the care and use of laboratory animals available. American Physiological Society. Physiologist 39: 199, 208-211, 1996
30. Bai XZ, Zhang JL, Liu Y, Zhang W, Li XQ, Wang KJ, Cao MY, Zhang JN, Han F, Shi JH and Hu DH: MicroRNA-138 aggravates inflammatory responses of macrophages by targeting SIRT1 and regulating the NF- $\kappa \mathrm{B}$ and AKT pathways. Cell Physio Biochem 49: 489-500, 2018.

31. Livak KJ and Schmittgen TD: Analysis of relative gene expression data using real-time quantitative PCR and the 2(-Delta Delta C(T)) method. Methods 25: 402-408, 2001.

32. Chen W, Ma X, Zhang P, Li Q, Liang X and Liu J: MiR-212-3p inhibits LPS-induced inflammatory response through targeting HMGB1 in murine macrophages. Exp Cell Res 350: 318-326, 2017.

33. Wang P, Zhang X, Li F, Yuan K, Li M, Zhang J, Li B and Liang W: MiR-130b attenuates vascular inflammation via negatively regulating tumor progression locus $2(\mathrm{Tpl} 2)$ expression. Int Immunopharmacol 51: 9-16, 2017.

34. Chithra MA, Ijinu TP, Kharkwal H, Sharma RK, Pushpangadan P and George V: Phenolic rich Cocos nucifera inflorescence extract ameliorates inflammatory responses in LPS-stimulated RAW264.7 macrophages and toxin-induced murine models. Inflammopharmacology: Jul 26, 2019 doi: 10.1007/s10787-019-00620-6 (Epub ahead of print).

35. Shou J, Kong X, Wang X, Tang Y, Wang C, Wang M, Zhang L, Liu Y, Fei C, Xue F, et al: Tizoxanide inhibits inflammation in LPS-Activated RAW264.7 macrophages via the suppression of NF- $\kappa B$ and MAPK activation. Inflammation 42: 1336-1349, 2019.

36. Ma A and Malynn BA: A20: Linking a complex regulator of ubiquitylation to immunity and human disease. Nat Rev Immunol 12: 774-785, 2012.

37. Huang L, Verstrepen L, Heyninck K, Wullaert A, Revets H, De Baetselier P and Beyaert R: ABINs inhibit EGF receptor-mediated NF-kappaB activation and growth of EGF receptor-overexpressing tumour cells. Oncogene 27: 6131-6140, 2008.

38. Pelegrin P, Barroso-Gutierrez C and Surprenant A: P2X7 receptor differentially couples to distinct release pathways for IL-1beta in mouse macrophage. J Immunol 180: 7147-7157, 2008.

39. Yonezawa Y, Miyashita T, Nejishima H, Takeda Y, Imai K and Ogawa H: Anti-inflammatory effects of olive-derived hydroxytyrosol on lipopolysaccharide-induced inflammation in RAW264.7 cells. J Vet Med Sci 80: 1801-1807, 2018.

40. Gao S, Wang Y, Li D, Guo Y, Zhu M, Xu S, Mao J and Fan G: TanshinoneIIA alleviates inflammatory response and directs macrophage polarization in lipopolysaccharide-stimulated RAW264.7 cells. Inflammation 42: 264-275, 2019.

41. Liu F, Zhang X, Ling P, Liao J, Zhao M, Mei L, Shao H, Jiang P, Song Z, Chen Q and Wang F: Immunomodulatory effects of xanthan gum in LPS-stimulated RAW264.7 macrophages. Carbohydr Polym 169: 65-74, 2017.

This work is licensed under a Creative Commons Attribution-NonCommercial-NoDerivatives 4.0 International (CC BY-NC-ND 4.0) License. 